\title{
Clinical assessment and management of auricular arteriovenous malformation: retrospective study
}

\author{
Do Gon Kim", \\ Hyun Geun Cho', \\ Jeong Yeop Ryu', \\ Joon Seok Lee ${ }^{1}$, \\ Seok Jong Lee ${ }^{2,3}$, \\ Jong Min Lee ${ }^{2,4}$, \\ Sang Yub Lee ${ }^{2,4}$, \\ Seung Huh ${ }^{2,5}$, \\ Ji Yoon Kim ${ }^{2,6}$, \\ Ho Yun Chung ${ }^{1,2,7,8}$ \\ ${ }^{1}$ Department of Plastic and \\ Reconstructive Surgery, School of \\ Medicine, Kyungpook National \\ University, Daegu; ${ }^{2}$ Vascular Anomalies \\ Center, Kyungpook National University \\ Hospital, Daegu; Departments of \\ ${ }^{3}$ Dermatology, ${ }^{4}$ Radiology, ${ }^{5}$ Surgery, and \\ ${ }^{6}$ Pediatrics, School of Medicine, \\ Kyungpook National University, Daegu; \\ ${ }^{7}$ CMRI and ${ }^{8}$ BK21 FOUR KNU \\ Convergence Educational Program of \\ Biomedical Sciences for Creative Future \\ Talents, School of Medicine, Kyungpook \\ National University, Daegu, Korea
}

\begin{abstract}
Background: Arteriovenous malformation (AVM) which is a high-blood-flow lesion with connections between arteries and veins without an intervening capillary bed, is difficult to manage. The ear is the second most common site of extracranial AVM. However, studies regarding the management of this condition remain lacking. The purpose of this study was to share managing experiences in our center and to investigate the treatment effect through a retrospective analysis of cases.

Methods: Among 265 patients with AVM treated in our vascular anomalies center between January 2008 and January 2021, 10 patients with auricular AVM were included in the study to investigate the lesion distribution, clinical stage, and treatment methods by performing a retrospective evaluation.

Results: Among 10 patients, five patients had AVMs distributed in the upper half of the ear, one patient in the lower half of the ear, and four patients in whole ear, respectively. Seven patients had Schobinger stage II, and three had stage III. One patient received surgical treatment only, four patients received sclerotherapy only, and five patients received both surgical treatment and sclerotherapy. The posttreatment status was checked as controlled in two patients, improved in seven patients, persistent in one patient. There were no worsening patients.

Conclusion: Auricular AVM is a disease that is difficult to manage by one specific department, thus requiring a collaborative management effort from multidisciplinary team.

Abbreviations: AVM, arteriovenous malformation; CT, computed tomography; D-USG, Doppler ultrasonography; $E$, resection with embolization; $M R I$, magnetic resonance imaging; PAA, posterior auricular artery; $\mathrm{R}$, surgical resection without embolization and reconstruction; $\mathrm{S}$, sclerotherapy; STA, superficial temporal artery; VAC, vascular anomalies center.
\end{abstract}

Keywords: Arteriovenous malformation / AVM protocol / Multidisciplinary communications

\section{INTRODUCTION}

Vascular anomaly is a collective name for abnormalities that develop during vascular development, so it is difficult to classify

Correspondence: Ho Yun Chung

Department of Plastic and Reconstructive Surgery, School of Medicine, Kyungpook National University, 130 Dongdeok-ro, Jung-gu, Daegu 41944, Korea E-mail: hy-chung@knu.ac.kr

This work was supported by the National Research Foundation of Korea (NRF) grant funded by the Korea government (MSIT) (No. 2020R1A2C2009496).

This article was presented as the 11th Research \& Reconstruction Forum on May 8, 2001, in Busan, Korea.

Received May 11, 2021 / Revised June 2, 2021 / Accepted June 20, 2021 because of its extensive symptoms and characteristics. It is a disorder of the endothelium and surrounding cells that affect the vasculature and surrounding structures. Mulliken and Glowacki divided vascular anomaly into hemangiomas and vascular malformations by using a biological classification system $[1,2]$.

Arteriovenous malformation (AVM) is vascular anomaly which is high-blood-flow lesions with connections between arteries and veins without an intervening capillary bed. In embryogenesis, AVM results from an error in vascular development, so it shows an arterial-to-venous circulation by forming a 
direct shunt through a fistula or nidus. The most common sites of extracranial AVM are the head and neck, followed by the limbs, trunk, and viscera [3]. Although AVM exists from birth, it may not be noticeable until childhood. AVM features a pinkred cutaneous stain with a palpable thrill or bruit, and distinguishing AVM from capillary malformations and hemangiomas is important. Arteriovenous shunt flow hinders the capillary oxygen delivery, causes ischemia, and results in complications such as pain, ulceration, bleeding, and congestive heart failure.

AVM is not a static malformation and progresses over time or may recur after treatment. Therefore, the treatment goal for AVM is usually controlling the progression of AVM by using multi-treatment methods such as sclerotherapy, embolization, and resection.

Extracranial AVMs in the head and neck are relatively rare. Auricular AVMs are the second most common after those in the cheek. Only few studies have been conducted on AVMs of the ear [4]. In 2005, Wu et al. [5] published a study with 41 patients with auricular AVM. The progression was assessed according to treatment method by dividing them into observation, ligation, embolization alone, and amputation groups. No improvement was observed in the observation and ligation groups. Of the 20 patients in the amputation group, 16 showed controlled findings and three showed improved findings. In 2009, Whitty et al. [6] published a case report on the treatment of AVM of the external ear in a 15-year-old patient. The AVM was excised, with no evidence of recurrence found during follow-up. In 2016, Kim et al. [7] published a study on embolization using trans-femoral cerebral angiography for an AVM of the ear in a 60-year-old male patient. In 2 weeks, after the necrotic skin boundary became distinctive, an excision operation was performed. In 2018, Gupta et al. [8] published a study in which preoperative coil embolization was performed for a huge AVM of the ear in a 24-year-old male patient, and ear reconstruction was performed using a split-thickness skin graft.

For the management for vascular anomalies, our hospital operates a vascular anomalies center (VAC), where plastic surgeons, intervention radiologists, dermatologists, vascular surgeons, and pediatrics participate. Patient consultations are conducted by one department or a multidisciplinary team. Before the multidisciplinary consultation, one physician takes charge of the patient and prepares the content of the interview questionnaires. Upon completion of the treatment plan from the multidisciplinary consultation, the patient undergoes consultations with a specialist from each field and then undergoes treatment. After the treatment, the next treatment plan is discussed during a multidisciplinary consultation in the VAC. Through
Table 1. Schobinger staging system for arteriovenous malformations

\begin{tabular}{ll}
\hline Stage & \multicolumn{1}{c}{ Features } \\
\hline I (quiescence) & Cutaneous blush or warmth \\
II (expansion) & Bruit, audible pulsation, and enlarging lesion \\
III (destruction) & Pain, ulceration, bleeding, and infection \\
IV (decompensation) & Cardiac failure \\
\hline
\end{tabular}

these sequential processes, we reviewed clinical data of auricular AVM patients and suggest the treatment protocol in our center.

\section{METHODS}

Among 265 patients who were diagnosed as having AVM radiologically and received treatment in our VAC between 2008 and 2021, 10 patients who were diagnosed as having auricular AVM were included in this retrospective study.

By reviewing the patients' charts, information on age, extent and side of the lesion, and signs and symptoms as designated by the Schobinger clinical staging system was obtained (Table 1). Findings from radiographic investigations, including Doppler ultrasonography, magnetic resonance imaging, computed tomography (CT), and angiography, were reviewed by two interventional radiologists. Available angiograms were studied, and the extent and distribution of the affected vascular territories were correlated to the clinical findings.

The patients received sclerotherapy, resection with/without preoperative embolization, or both. Surgery was performed by one plastic surgeon. After partial or complete AVM resection, primary closure, skin graft placement, otoplasty, or local flap placement were accomplished [9].

Four months after each treatment, the posttreatment status was evaluated by dividing into four stages as follows: stage 1 , controlled, no residual lesion; stage 2 , improved, with a residual lesion but without reexpansion; stage 3, persistent or stable, neither improved nor worsened; stage 4, recurred or worsened, aggravated signs and symptoms [5]. The further treatment plan was decided after the multidisciplinary consultation during the VAC visit.

\section{RESULTS}

Table 2 presents the summary of the cases of auricular AVM. The patients included were one man and nine women, with a mean age at presentation of 26 years (range, 6-60 years) and mean follow-up period of 5 years (range, 1-11 years). The lesions were localized in the upper auricle in four patients and the 
Table 2. Summary of the patient characteristics, clinical and radiological findings, and treatment

\begin{tabular}{|c|c|c|c|c|c|c|c|}
\hline Patient no. & Sex (yr) & $\begin{array}{c}\text { Age at initial } \\
\text { presentation (yr) }\end{array}$ & Location of the lesion & Side & Present at birth & $\begin{array}{l}\text { Palpable bruit or thrill or } \\
\text { warmth }\end{array}$ & Treatment \\
\hline 1 & Female & 28 & Upper auricle & Right & Yes & Yes & S\#1, R\#1 \\
\hline 2 & Female & 24 & Upper auricle & Left & Yes & Yes & $\mathrm{S} \# 1$ \\
\hline 3 & Female & 14 & Auricle and scalp & Left & Yes & Yes & S\#1, E\#1 \\
\hline 4 & Female & 6 & Upper auricle & Right & Yes & No & S\#1 \\
\hline 5 & Female & 27 & Auricle & Right & No & Yes & S\#2 \\
\hline 6 & Female & 20 & Auricle & Right & Yes & Yes & S\#1, E\#1 \\
\hline 7 & Male & 24 & Upper auricle & Left & No & Yes & $E \# 1, R \# 2$ \\
\hline 8 & Female & 60 & Auricle, cheek, and scalp & Left & No & Yes & S \#4, R \#1 \\
\hline 9 & Female & 46 & Ear lobe and chin & Left & No & Yes & $S \# 3, R \# 4$ \\
\hline 10 & Female & 31 & Tragus and preauricular area & Right & Yes & Yes & S\#4 \\
\hline
\end{tabular}

S, sclerotherapy; R, surgical resection without embolization and reconstruction; E, surgical resection with embolization.

Table 3. The location of auricular arteriovenous malformation and related feeding artery

\begin{tabular}{lcccccc}
\hline \multirow{2}{*}{ Feeding artery } & \multicolumn{4}{c}{ Location } & \multirow{2}{*}{ Total } \\
\cline { 2 - 5 } & Helix & Tragus & Ear lobe & Whole ear & \\
\hline Superficial temporal artery & 4 & 1 & - & 4 & 9 \\
Posterior auricular artery & - & - & 1 & 4 & 5 \\
\hline
\end{tabular}

Table 4. Summary of treatment outcomes

\begin{tabular}{lclcl}
\hline Patient no. & $\begin{array}{c}\text { Schobinger } \\
\text { stage }\end{array}$ & Treatment & $\begin{array}{c}\text { Posttreatment } \\
\text { stage }^{\text {a) }}\end{array}$ & \multicolumn{1}{c}{ Plan } \\
\hline 1 & $\|$ & S\#1, R\#1 & 2 & Conserve \\
2 & II & S\#1 & 2 & Conserve \\
3 & $\|$ & S\#1, E\#1 & 1 & Conserve \\
4 & II & S\#1 & 1 & Conserve \\
5 & II & S\#2 & 2 & Conserve \\
6 & I & S\#1, E\#1 & 2 & Conserve \\
7 & $\|$ & E\#1, R\#2 & 2 & Conserve \\
8 & II & S\#4, R\#1 & 2 & Conserve \\
9 & $\|$ & S\#3, R\#4 & 2 & Conserve \\
10 & $\|$ & S\#4 & 3 & Lost to follow-up \\
\hline
\end{tabular}

S, sclerotherapy; R, surgical resection without embolization and reconstruction; $E$, surgical resection with embolization.

a)Posttreatment stage: 1, controlled, no residual lesion; 2, improved, with a residual lesion but without reexpansion; 3 , persistent or stable, neither improved nor worsened; 4, recurred or worsened, aggravated signs and symptoms.

earlobe in one patient. In four patients, the lesions were distributed to the entire auricle and its surrounding tissues, and one patient was found to have lesions in the tragus and preauricular area (Table 3). Of the 10 patients, five had lesions in the right ear and five had lesions in the left ear. In six of the 10 patients, the lesion was found at birth. Nine patients showed a palpable bruit or thrill, or warmth at the time of initial hospital visit. Among the 10 patients, seven had Schobinger classification stage II and three had stage III before the start of treatment. Four patients received sclerotherapy only, one patient received surgical treat- ment only, and five patients underwent both sclerotherapy and surgical excision. All patients had showed symptoms such as bruit, thrill, and pain when starting treatment. After treatment, the symptoms disappeared in nine patients, AVM disappeared completely in two patients and did not recur, and it was well controlled in seven patients. It was checked as remaining unreduced in one patient (Table 4, Figs. 1-3).

\section{DISCUSSION}

AVM was first reported by John Bell in 1815 using the term "aneurysm by anastomosis." AVM is a pathological communication between arteries and veins via blood vessels such as a dilated capillary without interposition of the arteriole [10]. Collateral formation is consequently facilitated, and regional blood flow is increased at the peripheral level. Dilated, lengthened, and tortuous feeding arteries lead to the formation of a thick blood vessel wall after fibrous degeneration. The draining vein becomes hypertrophic, and increased intraluminal pressure results in a prominent muscular coat.

Schobinger classified AVM into four stages as follows: stage I (quiescence) features warm and discolored skin; stage II (expansion) features bruit, pulsation, and swelling; stage III (destruction) features pain, ulceration, and bleeding; and stage IV (decompression) features cardiac failure. The symptom and presenting sign are associated with the AVM stage. Although most AVMs are asymptomatic, they may cause symptoms such as severe pain and bleeding. Pulsation (51.2\%), bleeding (41.5\%), and pain (29.3\%) are the most common symptoms. In cases of AVM of the ear, hearing can also deteriorate because of the audible bruit [5].

The developmental origins of the upper ear, including the helix and lower ear, which consists of the ear lobe, are different. The upper ear is developed from the first pharyngeal arch and 

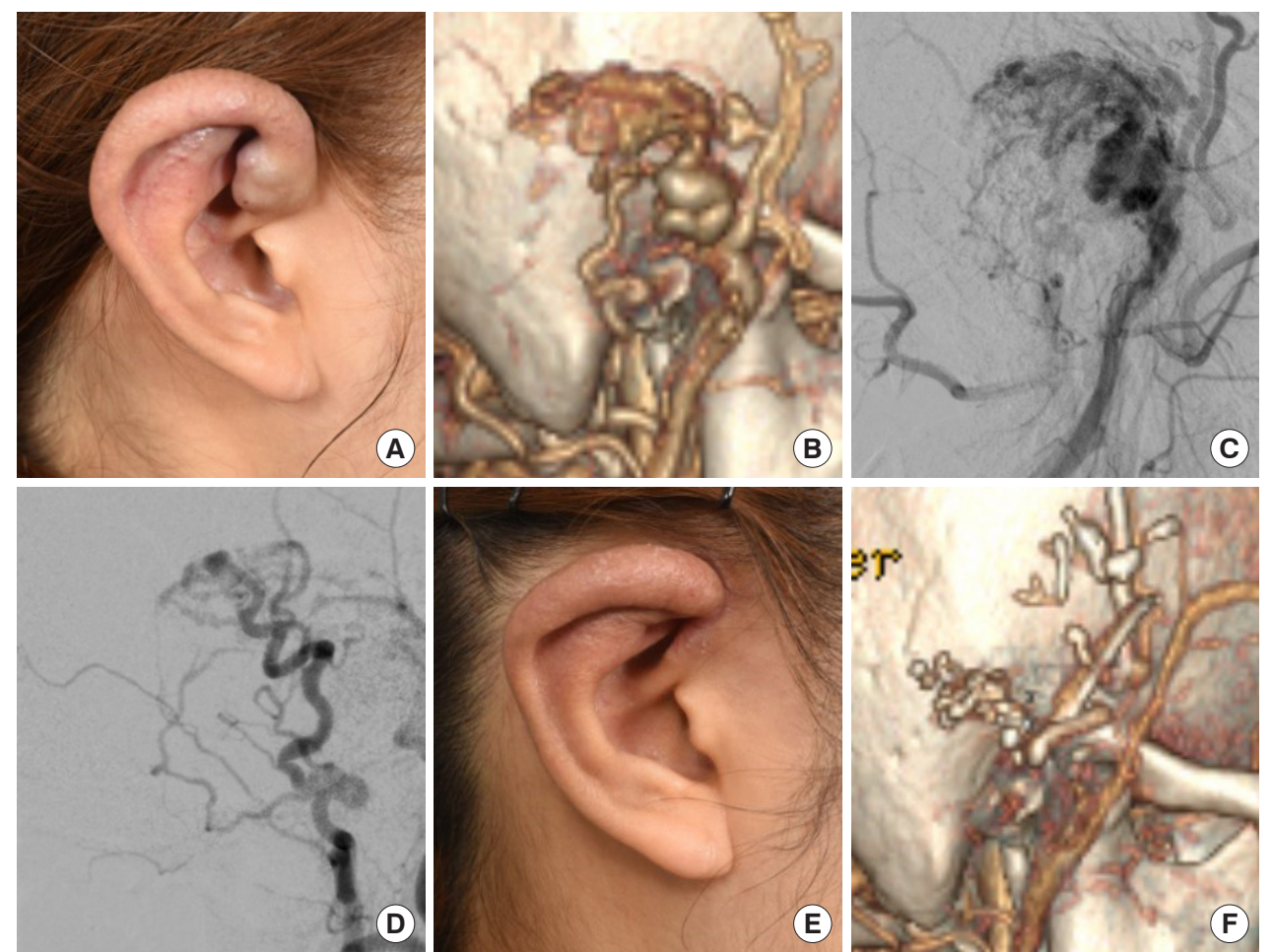

Fig. 1. Patient no. 1. A 28-year-old female patient with AVM in the upper auricle. the Schobinger stage II. (A, B) Preoperative clinical and CT findings. Direct puncture sclerotherapy was performed using a glue-and-lipiodol mixture. (C) Before sclerotherapy. (D) After sclerotherapy. After resection surgery, most of the AVM was removed, but the residual AVM was judged as posttreatment status stage 2. (E, F) Postoperative clinical and CT findings. AVM, arteriovenous malformation; CT, computed tomography.
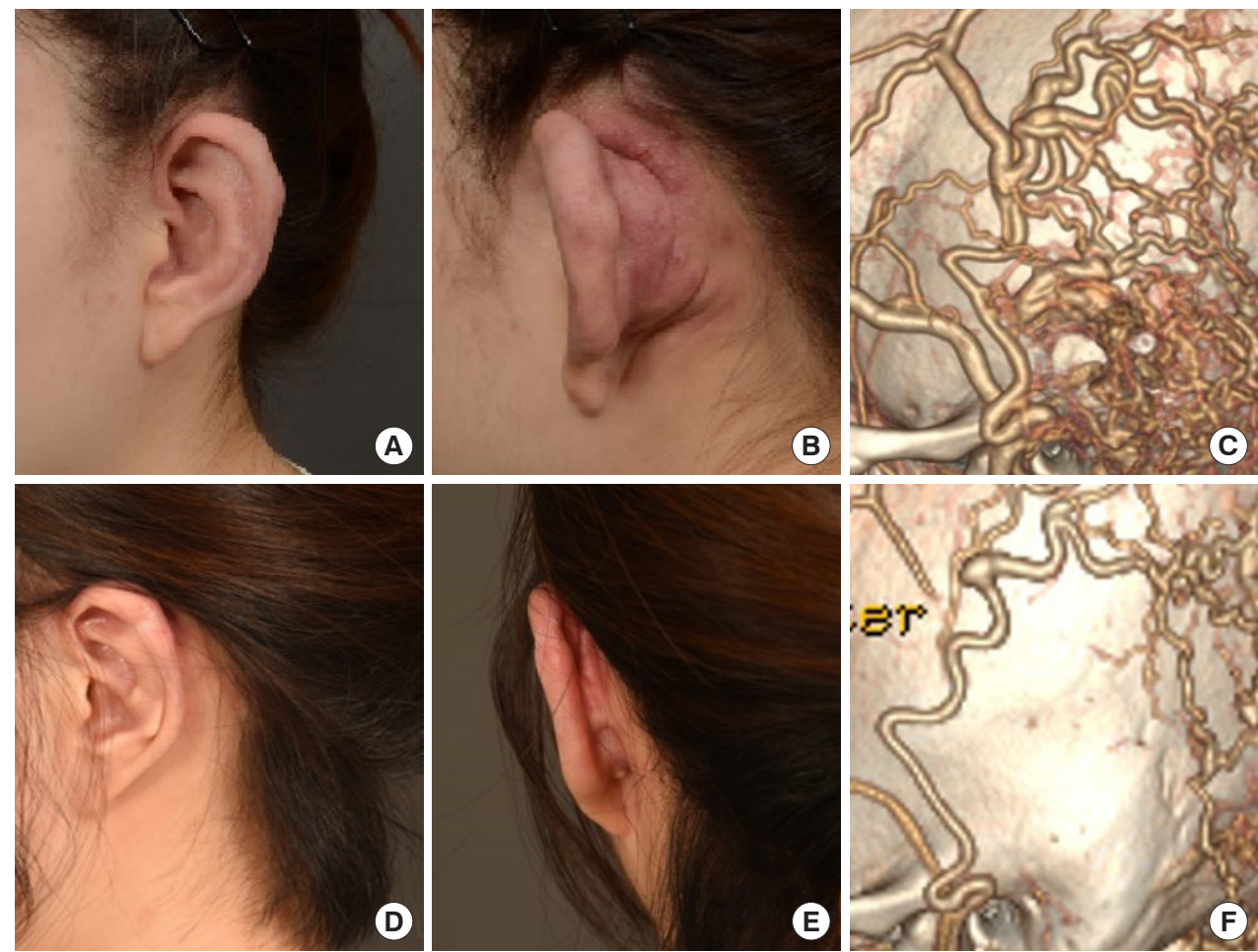

Fig. 2. Patient no. 3. A 14-year-old female patient with AVM in the upper auricle and scalp. the Schobinger stage II with no distinctive symptoms of diffuse scalp AVM. (A-C) Preoperative clinical and CT findings. Resection with embolization and trans-arterial sclerotherapy with ethanol were performed. Symptoms were resolved with no residual AVM of the ear, confirming posttreatment status stage 1. (D-F) Posttreatment clinical and CT findings. AVM, arteriovenous malformation; CT, computed tomography. 

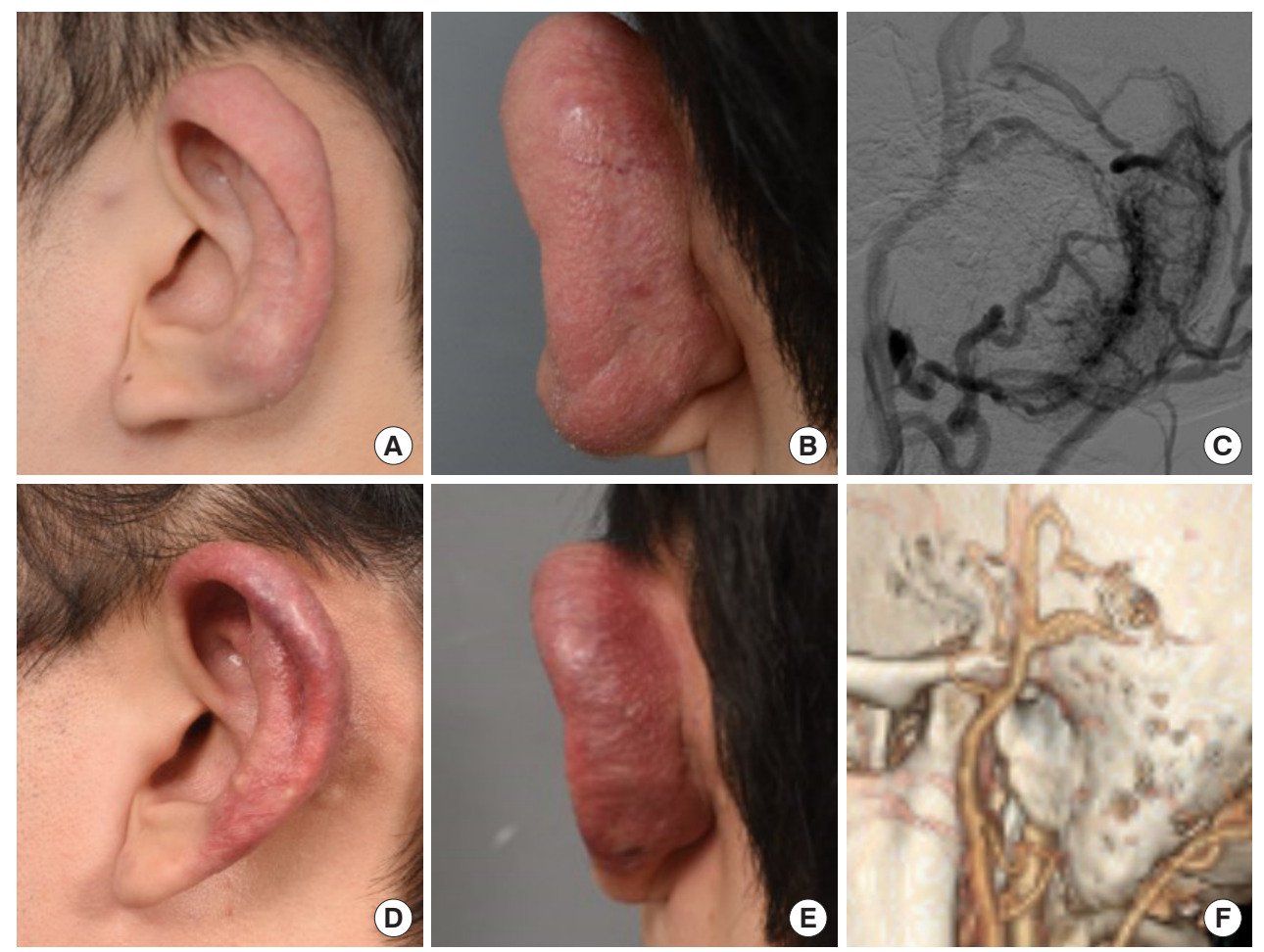

Fig. 3. Patient no. 7. A 24-year-old male patient with AVM in the upper auricle. the Schobinger stage II . (A-C) Preoperative clinical and angiography findings. After 1 year from first resection surgery, the lesion reexpanded. The posttreatment status was stage 4 . After multiple surgeries with embolization and reduction otoplasty, the symptoms were mostly improved, with a posttreatment status of stage 2. (D-F) Posttreatment clinical and CT findings. AVM, arteriovenous malformation; CT, computed tomography.

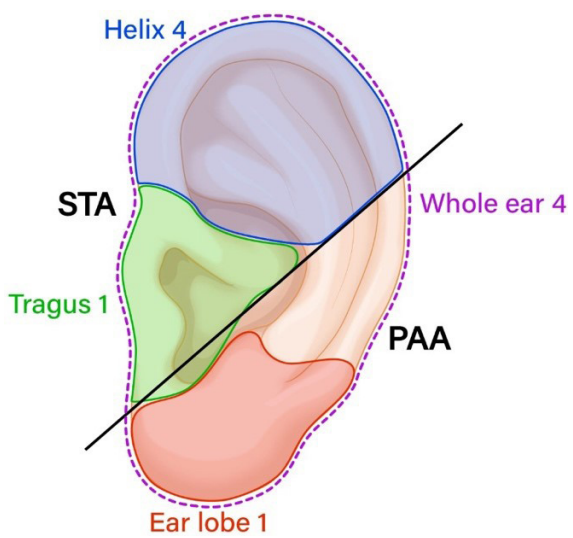

Fig. 4. The schematic diagram of ear development territories and auricular arteriovenous malformation distribution. The external ear develops from the anterior hillocks (first pharyngeal arch) and posterior hillocks (second pharyngeal arch). The schematic diagram depicts the vascular distribution of the auricle and the number of patients. STA, superficial temporal artery; PAA, posterior auricular artery.

receives blood supply from the posterior auricular artery. This may be used as reference for sclerotherapy. Patients with AVM in the helix, tragus, and whole ear account for $90 \%$ of our study patients. AVMs in the second pharyngeal arch, including the ear lobe, occur in $50 \%$ of the total patients, which implies that auricular AVM can occur more in case of development from the first pharyngeal arch origin than the second pharyngeal arch origin (Fig. 4) [5].

As treatments for AVM, embolization, sclerotherapy, and resection surgery have been used. Preoperative embolotherapy/ sclerotherapy is useful for solidifying AVM lesions, as it reduces the incidence of bleeding and defines the border of the lesion. Especially in pediatric patients, if the AVM is small and has no symptoms, treatment may not be necessary. In cases with symptoms, complete excision may be considered the treatment of choice. However, further enlargement may result from the formation of a new collateral circulation, so ligation of the arterial vessel alone and partial excision must be avoided.

As the ear is exposed and surgical treatment may cause ear deformities and excessive scar formation, sclerotherapy is preferred to surgical excision. However, symptom relief from mass effect could not be expected because single sclerotherapy cannot reduce the AVM volume effectively. If complete excision is possible through a surgical resection, both the mass and debulking effects may be achievable.

Although many researchers have studied the treatment methods for AVM and their effects, a unified treatment strategy has not been established yet. Therefore, a treatment plan should be 
established by taking into consideration the symptoms of each patient; the location, depth, size, and type of lesion; and side effects. We set up basic treatment protocol for auricular AVM. Surgical treatment was considered in cases for which total resection was possible, and the incidence of bleeding was reduced with preoperative embolization in cases with high bleeding risks. Sclerotherapy was considered for cases of unresectable AVM. Alternatively, depending on the patient's condition, sclerotherapy was first considered.

In our study, four patients received sclerotherapy only, one received surgical treatment only, and five received both sclerotherapy and surgical excision. The treatment plans for the patients were decided through multidisciplinary consultations. At their visit to the VAC, seven of the 10 patients had stage II AVM and three had stage III AVM according to the Schobinger staging system. Treatments were performed in accordance with the patients' conditions, which were posttreatment stage 1 in two patients, stage 2 in seven patients, and stage 3 in one patient. The further treatment plan was decided in accordance with the posttreatment stage. Nine patients underwent conservative treatment, and one patient was lost to follow-up (Table 4).

Ear amputation is considered for total resection of auricular AVM. If amputation is performed as AVM treatment, several staged ear reconstruction are required; otherwise, patient satisfaction is decreased with regard to the aesthetic outcome. Thus, in our center, we do our best to minimize ear deformities as much as possible with sclerotherapy and AVM resection.

When auricular AVM was diagnosed upon visit to the VAC, if the lesion was well localized and total resection surgery was possible, resection surgery was performed. In other cases, sclerotherapy was the first consideration. Surgery was conducted after embolization preoperatively in cases with high bleeding risks; otherwise, only surgery was conducted. Four months after single treatment, the patients were reevaluated using Doppler ultrasonography, CT angiography, and so on. In cases of posttreatment stage 1, to improve the aesthetic outcome and patient satisfaction, laser treatment and otoplasty could be conducted. For cases of posttreatment stage 2 or higher, either further treatment or regular follow up with radiologic examination need to be considered (Fig. 5).

A multidisciplinary approach is necessary in the diagnosis and treatment of AVM because the existing single-treatment system might easily lead to misdiagnosis or wrong treatment [11]. In 2002, Very et al. [12] compared the history of diagnosis in other medical institutes with that in a medical center specializing in vascular tumors and malformations, and found that the accurate diagnosis rate was only $37 \%$. In 2004, Mathes et al. [13] published that only $22.4 \%$ of all diagnoses matched in patients

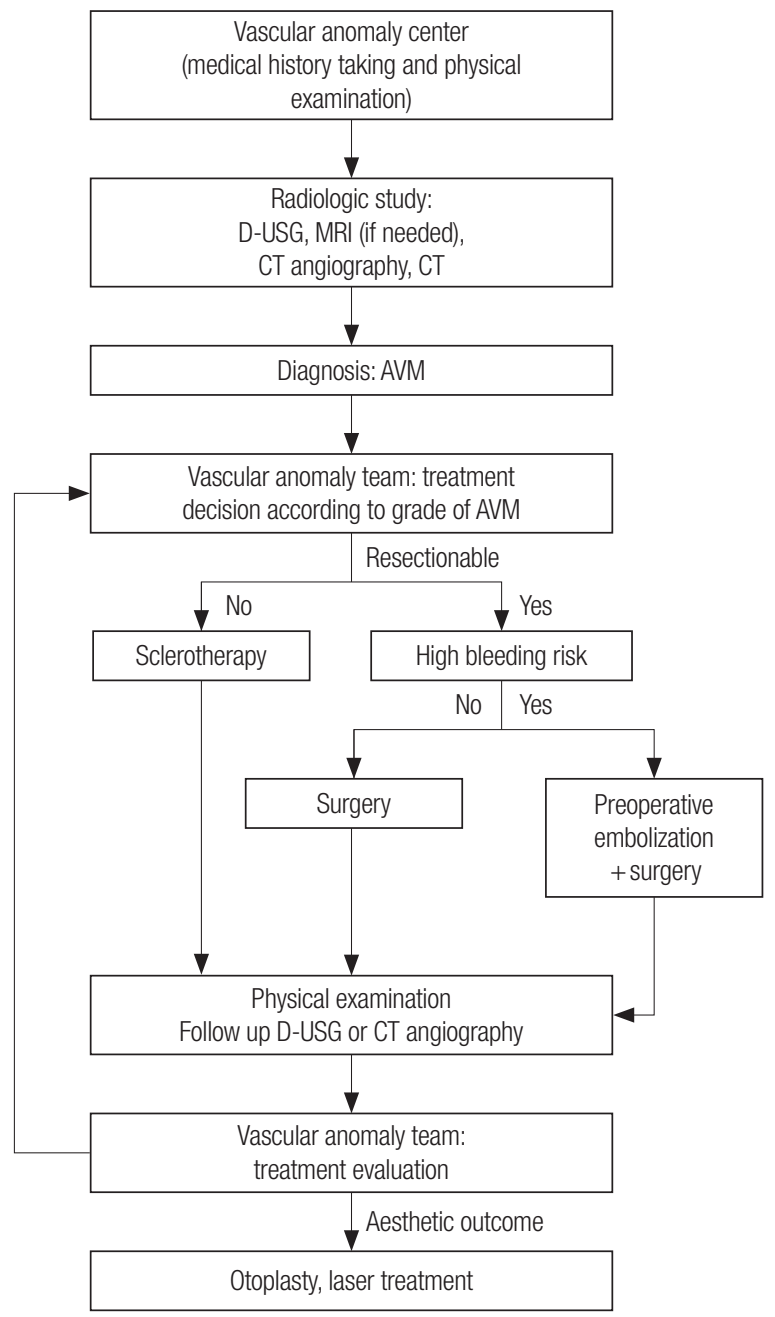

Fig. 5. The algorithm used at our vascular anomalies center for the diagnosis and treatment of patients with auricular AVMs. D-USG, Doppler ultrasonography; MRI, magnetic resonance imaging; CT, computed tomography; AVM, arteriovenous malformation.

who visited the VAC of California University in San Francisco. In addition, a multidisciplinary approach including specialists from various fields may provide broad treatment methods for patients and medical staff to choose from. Consequently, a multidisciplinary approach to AVM enables a more accurate diagnosis and effective treatment than the existing single-treatment system. A protocol that can be used as a guideline should be used as a reference, but treatment methods should be decided flexibly in accordance with the patient's condition and characteristics.

\section{NOTES}

\section{Conflict of interest}

No potential conflict of interest relevant to this article was reported. 


\section{Ethical approval}

The study was approved by the Institutional Review Board of Kyungpook National University Hospital (IRB No. 2021-02-013) and performed in accordance with the principles of the Declaration of Helsinki. Written informed consent was obtained.

\section{Patient consent}

The patients provided written informed consent for the publication and the use of their images.

\section{ORCID}

$\begin{array}{ll}\text { Do Gon Kim } & \text { https://orcid.org/0000-0002-7835-3004 } \\ \text { Hyun Geun Cho } & \text { https://orcid.org/0000-0003-3851-0623 } \\ \text { Jeong Yeop Ryu } & \text { https://orcid.org/0000-0003-2812-5051 } \\ \text { Joon Seok Lee } & \text { https://orcid.org/0000-0002-1580-0487 } \\ \text { Seok Jong Lee } & \text { https://orcid.org/0000-0002-6131-632X } \\ \text { Jong Min Lee } & \text { https://orcid.org/0000-0002-4163-913X } \\ \text { Sang Yub Lee } & \text { https://orcid.org/0000-0001-8529-8229 } \\ \text { Seung Huh } & \text { https://orcid.org/0000-0002-0275-4960 } \\ \text { Ji Yoon Kim } & \text { https://orcid.org/0000-0002-5577-6629 } \\ \text { Ho Yun Chung } & \text { https://orcid.org/0000-0001-7359-3044 }\end{array}$

\section{Author contribution}

Investigation: DGK, HGC, JSL. Supervision: HYC. Data curation: DGK, JYR. Formal analysis: DGK. Project administration: SJL, JML, SYL, SH, JYK. Writing - original draft: DGK. Writing - review \& editing: DGK, HGC, HYC.

\section{REFERENCES}

1. Mulliken JB, Glowacki J. Hemangiomas and vascular malformations in infants and children: a classification based on endothelial characteristics. Plast Reconstr Surg 1982;69:412-22.

2. Ryu JY, Lee JH, Lee JS, Lee JW, Lee SJ, Lee JM, et al. Combined treatment of surgery and sclerotherapy for sinus pericranii. Arch Craniofac Surg 2020;21:109-13.
3. Kim JB, Lee JW, Choi KY, Yang JD, Cho BC, Lee SJ, et al. Clinical characteristics of arteriovenous malformations of the head and neck. Dermatol Surg 2017;43:526-33.

4. Lee JW, Chung HY. Vascular anomalies of the head and neck: current overview. Arch Craniofac Surg 2018;19:243-7.

5. Wu JK, Bisdorff A, Gelbert F, Enjolras O, Burrows PE, Mulliken JB. Auricular arteriovenous malformation: evaluation, management, and outcome. Plast Reconstr Surg 2005;115:985-95.

6. Whitty LA, Murray JD, Null WE, Elwood ET, Jones GE. An arteriovenous malformation of the external ear in the pediatric population: a case report and review of the literature. Can J Plast Surg 2009;17:e45-7.

7. Kim SH, Han SH, Song Y, Park CS, Song JJ. Arteriovenous malformation of the external ear: a clinical assessment with a scoping review of the literature. Braz J Otorhinolaryngol 2017; 83:683-90

8. Gupta A, Gupta S, Kumar A, Bhattacharaya S, Jha M, Tiwari V. High-flow vascular malformation of ear: a case report. World J Plast Surg 2018;7:256-60.

9. Ryu JY, Eo PS, Lee JS, Lee JW, Lee SJ, Lee JM, et al. Surgical approach for venous malformation in the head and neck. Arch Craniofac Surg 2019;20:304-9.

10. Prasad KC, Prasad SC, Shahul S. Spontaneous arteriovenous malformation of the ear. Indian J Otolaryngol Head Neck Surg 2011;63(Suppl 1):19-22.

11. Horbach SER, Ubbink DT, Stubenrouch FE, Koelemay MJW, van der Vleuten CJM, Verhoeven BH, et al. Shared decisionmaking in the management of congenital vascular malformations. Plast Reconstr Surg 2017;139:725e-734e.

12. Very M, Nagy M, Carr M, Collins S, Brodsky L. Hemangiomas and vascular malformations: analysis of diagnostic accuracy. Laryngoscope 2002;112:612-5.

13. Mathes EF, Haggstrom AN, Dowd C, Hoffman WY, Frieden IJ. Clinical characteristics and management of vascular anomalies: findings of a multidisciplinary vascular anomalies clinic. Arch Dermatol 2004;140:979-83. 